\title{
Varieties with at most cubic growth
}

\author{
S. Mishchenko ${ }^{1}$ \\ Department of Applied Mathematics \\ Ulyanovsk State University \\ Ulyanovsk 432970, Russia \\ A. Valenti ${ }^{1, *}$ \\ Dipartimento di Energia, ingegneria dell'Informazione e Modelli Matematici \\ Università di Palermo \\ 90128 Palermo, Italy
}

\begin{abstract}
Let $\mathcal{V}$ be a variety of non necessarily associative algebras over a field of characteristic zero. The growth of $\mathcal{V}$ is determined by the asymptotic behavior of the sequence of codimensions $c_{n}(\mathcal{V}), n=1,2, \ldots$, and here we study varieties of polynomial growth. We classify all possible growth of varieties $\mathcal{V}$ of algebras satisfying the identity $x(y z) \equiv 0$ such that $c_{n}(\mathcal{V})<C n^{\alpha}$, with $1<\alpha<3$, for some constant $C$. We prove that if $1<\alpha<2$ then $c_{n}(\mathcal{V}) \leq C_{1} n$, and if $2<\alpha<3$, then $c_{n}(\mathcal{V}) \leq C_{2} n^{2}$, for some constants $C_{1}, C_{2}$.
\end{abstract}

Keywords: Varieties, codimension growth

2010 MSC: Primary 17A30 16R10, Secondary 16P90

\section{Introduction}

Let $F$ be a field of characteristic zero and $F\{X\}$ the free non associative algebra on a countable set $X$ over $F$. Let $\mathcal{V}$ be a variety of non necessarily associative algebras and $\operatorname{Id}(\mathcal{V})$ be the $T$-ideal of identities of $\mathcal{V}$. In characteristic zero without loss of generality one can study the multilinear identities of $\mathcal{V}$ and a natural and well established way of measuring the identities of $\mathcal{V}$ is through the study of the asymptotic behavior of its sequence of codimensions $c_{n}(\mathcal{V}), n=1,2, \ldots$. More precisely, for every $n \geq 1$ let $P_{n}$ be the space of multilinear polynomials in the variables $x_{1}, \ldots, x_{n}$. Since char $F=0$, the T-ideal $\operatorname{Id}(\mathcal{V})$ is determined by the multilinear polynomials it contains; hence the relatively free algebra $F\{X\} / I d(\mathcal{V})$ is determined by the sequence of subspaces $\left\{P_{n} /\left(P_{n} \cap I d(\mathcal{V})\right)\right\}_{n \geq 1}$. The integer $c_{n}(\mathcal{V})=\operatorname{dim} P_{n} /\left(P_{n} \cap I d(\mathcal{V})\right)$ is called the $n$-th codimension of $\mathcal{V}$ and the growth function determined by the sequence of integers $\left\{c_{n}(\mathcal{V})\right\}_{n \geq 1}$ is the growth of the variety $\mathcal{V}$.

If $\mathcal{V}=\operatorname{var}(A)$ is the variety generated by an algebra $A$, then we write $\operatorname{Id}(\mathcal{V})=\operatorname{Id}(A)$ and $c_{n}(A)=c_{n}(\mathcal{V})$.

The first result on the asymptotic behavior of $c_{n}(\mathcal{V})$ is due to Regev $([16])$. He proved that if $\mathcal{V}$ is a non-trivial variety of associative algebras, then the sequence of codimensions

\footnotetext{
* Corresponding author

Email addresses: mishchenkosp@mail.ru (S. Mishchenko), angela.valenti@unipa.it (A. Valenti )

${ }^{1}$ The second author was partially supported by INDAM-GNSAGA of Italy.

Preprint submitted to Elsevier 
is exponentially bounded, i.e., there exist constants $\alpha, a>0$ such that $c_{n}(\mathcal{V}) \leq \alpha a^{n}$, for all $n$. In case $\mathcal{V}$ is a variety of non associative algebras, such sequence has a much more involved behavior and can have overexponential growth ([15]). Nevertheless for varieties of associative and Lie algebras, no intermediate growth (between polynomial and exponential) and no exponential growth between 1 and 2 is allowed $([9],[10],[11])$.

The exponential rate of growth of the sequence of codimensions of an associative algebra was determined in [5] and [6]. It was proved that for any associative PI-algebra $A$, the limit $\lim _{n \rightarrow \infty} \sqrt[n]{c_{n}(A)}$ exists and is a non-negative integer. In case of finite dimensional Lie algebra the same result was proved in [17]. This is not an expected behavior for Lie algebras, in fact in [18] was constructed an example of a Lie algebra whose sequence of codimensions grows exponentially but the rate of growth is not integer.

In this paper we consider varieties $\mathcal{V}$ of not necessarily associative algebras such that the sequence of codimensions is polynomially bounded, i.e., there exist constants $\alpha, t>0$ such that $c_{n}(\mathcal{V}) \leq \alpha n^{t}$, for all $n$. The asymptotic behavior of the codimensions of a unitary algebra was described by Drensky $([3])$. He proved that if $\mathcal{V}$ is a variety of associative or Lie algebras whose sequence of codimensions is polynomially bounded then the growth of the codimensions is exactly polynomial, i.e., there exist a positive integer $k$ and a constant $C$ such that $c_{n}(\mathcal{V})=C n^{k}+O\left(n^{k-1}\right)$, where $O\left(n^{k-1}\right)$ is a polynomial of degree $\leq k-1$.

In this paper we deal with the variety, $\mathcal{V}={ }_{2} \mathcal{N}$, of left nilpotent algebras of index two, that is the variety of algebras satisfying the identity

$$
x(y z) \equiv 0 .
$$

For this class of algebras in [14] the authors constructed a variety $\mathcal{W} \subset{ }_{2} \mathcal{N}$ such that for any $n \geq 25$

$$
([\sqrt{n}]-2) \frac{n(n-1)(n-5)}{6} \leq c_{n}(\mathcal{V}) \leq n^{3} \sqrt{n}+n^{2}(2 n+3 \sqrt{n})+n^{2} .
$$

In other words, the variety $\mathcal{W}$ has fractional polynomial growth between 3 and 4 , more precisely $\lim _{n \rightarrow \infty} \log _{n} c_{n}(\mathcal{V})=\frac{7}{2}$.

Motivated by this results in $([12],[13])$ we classified the growth of varieties of commutative and anticommutative algebras with at most quadratic growth. We proved that if $\mathcal{V}$ is a variety such that $c_{n}(\mathcal{V})<C n^{\alpha}$ with $0<\alpha<1$, then $c_{n}(\mathcal{V}) \leq 1$, for $n$ large. Moreover if $1<\alpha<2$, then either $\lim _{n \rightarrow \infty} \log c_{n}(\mathcal{V})=1$ or $c_{n}(\mathcal{V}) \leq 1$, for $n$ large.

The purpose of this paper is to prove that if $\mathcal{V}$ is the variety of algebras satisfying the identity $x(y z) \equiv 0$ and $c_{n}(\mathcal{V}) \leq C n^{\alpha}$ with $1<\alpha<2$, then $c_{n}(\mathcal{V})<C_{1} n$, for some constant $C_{1}$. Moreover if $c_{n}(\mathcal{V}) \leq C n^{\alpha}$, with $2<\alpha<3$, then $c_{n}(\mathcal{V})<C_{2} n^{2}$ for some constant $C_{2}$.

\section{Preliminaries}

Throughout $F$ will be a field of characteristic zero, $X=\left\{x_{1}, x_{2}, \ldots\right\}$ a countable set and $F\{X\}$ the free non associative algebra on $X$ over $F$. Let $\mathcal{V}$ be a variety and $\operatorname{Id}(\mathcal{V})=$ $\{f \in F\{X\} \mid f \equiv 0$ on $\mathcal{V}\}$ be the T-ideal of identities of $\mathcal{V}$. For every $n \geq 1$, let $P_{n}$ be the space of multilinear polynomials of $F\{X\}$ in the first $n$ variables $x_{1}, x_{2}, \ldots, x_{n}$. Since $\operatorname{char} F=0$, it is well known that the sequence of spaces $P_{n} \cap \operatorname{Id}(\mathcal{V}), n=1,2, \ldots$, carry all information about $I d(\mathcal{V})$. The symmetric group $S_{n}$ acts on $P_{n}$ by permuting variables: if $\sigma \in S_{n}, f\left(x_{1}, \ldots, x_{n}\right) \in P_{n}$

$$
\sigma f\left(x_{1}, \ldots, x_{n}\right)=f\left(x_{\sigma(1)}, \ldots, x_{\sigma(n)}\right) .
$$


The space $P_{n} \cap \operatorname{Id}(\mathcal{V})$ is invariant under this action and one studies the structure of $P_{n}(\mathcal{V})=P_{n} /\left(P_{n} \cap I d(\mathcal{V})\right)$ as an $S_{n}$-module. The $S_{n}$-character of $P_{n}(\mathcal{V})$, denoted $\chi_{n}(\mathcal{V})$, is called the $n$th cocharacter of $A$. Its degree $c_{n}(\mathcal{V})=\chi_{n}(\mathcal{V})(1)$ is the $n$th codimension of $\mathcal{V}$. By complete reducibility one writes

$$
\chi_{n}(\mathcal{V})=\sum_{\lambda \vdash n} m_{\lambda} \chi_{\lambda}
$$

where $\chi_{\lambda}$ is the irreducible $S_{n}$-character corresponding to the partition $\lambda$ of $n$ and $m_{\lambda} \geq 0$ is the multiplicity of $\chi_{\lambda}$ (see for instance [8] for the representation theory of the symmetric group).

Notice that in case $\mathcal{V}$ is a variety of associative algebras, for the multiplicities $m_{\lambda}$ we have that $m_{\lambda} \leq d_{\lambda}$, where $d_{\lambda}=\operatorname{deg} \chi_{\lambda}$ is the degree of the character $\chi_{\lambda}$. In the non associative case this inequality does not hold any more. For instance for the free non associative algebra $A=F\{X\}$ we have that, in $\chi_{n}(A), m_{\lambda}=C_{n} d_{\lambda}$ where $C_{n}$ is the $n$th Catalan number.

We next recall some basic properties of the representation theory of the symmetric group that we shall use in the sequel. Let $\lambda \vdash n$ and let $T_{\lambda}$ be a Young tableau of shape $\lambda \vdash n$. We denote by $e_{T_{\lambda}}$ the corresponding essential idempotent of the group algebra $F S_{n}$. Recall that $e_{T_{\lambda}}=\bar{R}_{T_{\lambda}} \bar{C}_{T_{\lambda}}$ where

$$
\begin{gathered}
\bar{R}_{T_{\lambda}}=\sum_{\sigma \in R_{T_{\lambda}}} \sigma, \\
\bar{C}_{T_{\lambda}}=\sum_{\tau \in C_{T_{\lambda}}}(\operatorname{sgn} \tau) \tau
\end{gathered}
$$

and $R_{T_{\lambda}}, C_{T_{\lambda}}$ are the row and column stabilizers of $T_{\lambda}$, respectively. Recall that if $M_{\lambda}$ is an irreducible $S_{n}$-submodule of $P_{n}(\mathcal{V})$ corresponding to $\lambda$, there exists a polynomial $f\left(x_{1}, \ldots, x_{n}\right) \in P_{n}$ and a tableau $T_{\lambda}$ such that $e_{T_{\lambda}} f\left(x_{1}, \ldots, x_{n}\right) \notin \operatorname{Id}(\mathcal{V})$.

In what follows we shall use also the representation theory of the general linear group. Let $m \geq 1$ and $U=\operatorname{span}_{F}\left\{x_{1}, \ldots, x_{m}\right\}$. The group $G L(U) \cong G L_{m}$ acts naturally on the left on the space $U$ and we can extend this action diagonally to get an action on $F_{m}\{X\}=$ $F\left\{x_{1}, \ldots, x_{m}\right\}$, the free algebra of rank $m$.

The space $F_{m}\{X\} \cap I d(\mathcal{V})$ is invariant under this action, hence

$$
F_{m}(\mathcal{V})=\frac{F_{m}\{X\}}{F_{m}\{X\} \cap I d(\mathcal{V})}
$$

inherits a structure of left $G L_{m}$-module. Let $F_{m, n}$ be the space of homogeneous polynomials of degree $n$ in the variables $x_{1}, \ldots, x_{m}$, then

$$
F_{m, n}(\mathcal{V})=\frac{F_{m, n}}{F_{m, n} \cap I d(\mathcal{V})}
$$

is a $G L_{m}$-submodule of $F_{m}(\mathcal{V})$ and we denote its character by $\psi_{n}(\mathcal{V})$. Write

$$
\psi_{n}(\mathcal{V})=\sum_{\lambda \vdash n} \bar{m}_{\lambda} \psi_{\lambda}
$$

where $\psi_{\lambda}$ is the irreducible $G L_{m}$-character associated to the partition $\lambda$ and $\bar{m}_{\lambda}$ is the corresponding multiplicity. In [1] and [2] it was proved that if the character $\chi_{n}(\mathcal{V})$ has the decomposition given in (1) then $m_{\lambda}=\bar{m}_{\lambda}$, for all $\lambda \vdash n$ whose corresponding diagram has height at most $m$. 
It is also known (see for instance [4, Theorem 12.4.12 ]) that any irreducible submodule of $F_{m, n}(\mathcal{V})$ corresponding to $\lambda$ is generated by a non-zero polynomial $f_{\lambda}$, called highest weight vector, of the form

$$
f_{\lambda}=\prod_{i=1}^{\lambda_{1}} S t_{h_{i}(\lambda)}\left(x_{1}, \ldots, x_{h_{i}(\lambda)}\right) \sum_{\sigma \in S_{n}} \alpha_{\sigma} \sigma
$$

where $\alpha_{\sigma} \in F$, the right action of $S_{n}$ on $F_{m, n}^{T}(A)$ is defined by place permutation, $h_{i}(\lambda)$ is the height of the $i$ th column of the diagram of $\lambda$ and

$$
S t_{r}\left(x_{1}, \ldots, x_{r}\right)=\sum_{\tau \in S_{r}}(\operatorname{sgn} \tau) x_{\tau(1)} \cdots x_{\tau(r)}
$$

is the standard polynomial of degree $r$ with a suitable arrangement of the parentheses. Recall that $f_{\lambda}$ is unique up to a multiplicative constant.

For a Young tableau $T_{\lambda}$, denote by $f_{T_{\lambda}}$ the highest weight vector obtained from (2) by considering the only permutation $\sigma \in S_{n}$ such that the integers $\sigma(1), \ldots, \sigma\left(h_{1}(\lambda)\right)$, in this order, fill in from top to bottom the first column of $T_{\lambda}, \sigma\left(h_{1}(\lambda)+1\right), \ldots, \sigma\left(h_{1}(\lambda)+h_{2}(\lambda)\right)$ the second column of $T_{\lambda}$, etc.

By [4, Proposition 12.4.14] we have that if

$$
\psi_{n}(\mathcal{V})=\sum_{\lambda \vdash n} \bar{m}_{\lambda} \psi_{\lambda}
$$

is the $G L_{m}$-character of $F_{m, n}(\mathcal{V})$, then $\bar{m}_{\lambda}$ is equal to the maximal number of linearly independent highest weight vectors $f_{T_{\lambda}}$ in $F_{m, n}(\mathcal{V})$.

\section{Classifying varieties $\mathcal{V}$ such that $c_{n}(\mathcal{V}) \leq C n^{\alpha}, 1<\alpha<2$}

Throughout this section we shall assume that $\mathcal{V}$ is the variety of left nilpotent algebras of index two, that is the variety of algebras satisfying the identity

$$
x(y z) \equiv 0
$$

such that $c_{n}(\mathcal{V}) \leq C n^{\alpha}$, for some $1<\alpha<2$, and for some constant $C$.

Our aim is to prove that for such variety $c_{n}(\mathcal{V})<C_{1} n$, for some constant $C_{1}$.

Notice that modulo the identity $x(y z) \equiv 0$ all non-zero monomials of the free algebra are left normed, i.e., are of the type $\left(\left(\left(x_{1} x_{2}\right) x_{3}\right) \ldots\right)$. Since we shall be working modulo such identity throughout we shall omit the parenthesis in left normed monomials, hence we shall write $\left(\left(\left(x_{1} x_{2}\right) x_{3}\right) \ldots x_{n}\right)=x_{1} x_{2} \ldots x_{n}$, and $x y^{2}$ for $x y y$.

In what follows we shall make use of the following lemma which was proved in [12].

Lemma 1. If $\lambda \vdash n$ is such that $\lambda \notin\left\{(n),\left(1^{n}\right),(n-1,1),\left(2,1^{n-2}\right)\right\}$ then $d_{\lambda} \geq \frac{1}{8} n^{2}$.

From the above lemma it follows that if $\lambda \vdash n$ is distinct from $(n),\left(1^{n}\right),(n-1,1),\left(2,1^{n-2}\right)$, then there exists $N>0$ such that for all $n \geq N$ we have that $d_{\lambda}>C n^{\alpha}$ and hence $m_{\lambda}=0$. We fix the integer $N$ from now on.

The following remark is obvious.

Remark 1. Let $\lambda \vdash n$. If either $\lambda=(n)$ or $\lambda=\left(1^{n}\right)$ then $m_{\lambda} \leq 1$. 
Let $\lambda \vdash n$ be a partition of $n$ and $f_{i}, i=1,2, \ldots, \operatorname{deg} \chi_{\lambda}$, be polynomials corresponding to the standard Young tableaux of shape $\lambda$ in $P_{n}$. For every $i$, let $T_{i}$ be the corresponding standard tableaux and denote by $g_{i}$ the polynomial obtained from $f_{i}$ by identifying with $x_{1}$ all variables corresponding to the first row of $T_{i}$, with $x_{2}$ all variables corresponding to the second row of $T_{i}$ and so on. Then, by [4, Proposition 12.4.14 ], $m_{\lambda}$ equals the dimension of the space spanned by all $g_{i}, 1 \leq i \leq \operatorname{deg} \chi_{\lambda}, \bmod \operatorname{Id}(\mathcal{V})$. In what follows we shall use this fact without mention it.

We shall adopt the convention of marking a set of alternating variables with the same symbol,$\sim$. For instance, in $\overline{x_{1}} y_{1} \overline{x_{2}} y_{2} \overline{x_{3}}$ stands for $\sum_{\sigma \in S_{3}}(\operatorname{sgn} \sigma) x_{\sigma(1)} y_{1} x_{\sigma(2)} y_{2} x_{\sigma(3)}$.

The following result concern the partition $\lambda=(n-1,1)$.

Proposition 1. If $\chi_{n}(\mathcal{V})=\sum_{\lambda \vdash n} m_{\lambda} \chi_{\lambda}$ and $n \geq N$ we have that

$$
m_{(n-1,1)} \leq 2 .
$$

Proof. Let $\lambda=(n-2,1,1) \vdash n$. For every $i=0, \ldots, n-3$, let

$$
f_{i}=\bar{x}_{1} x_{1}^{i} \bar{x}_{2} \bar{x}_{3} x_{1}^{n-i-3}
$$

be the left normed polynomials corresponding to the following standard tableaux

$$
T_{\lambda}^{(i)}=\begin{array}{c|c|c|c|c|c|c|}
\hline 1 & 2 & \ldots & i+1 & i+4 & \ldots & n \\
\hline i+2 & \multicolumn{7}{|l}{} \\
\cline { 1 - 1 } i+3 &
\end{array}
$$

Since if $n \geq N$, by Lemma $1, d_{\lambda}>C n^{\alpha}, 1<\alpha<2$, then it follows that, for every $i=0, \ldots, n-3$,

$$
f_{i} \equiv 0 \quad(\bmod . \operatorname{Id}(\mathcal{V}))
$$

Let consider the following substitution $x_{1}=z x_{1}+x_{1}$ then we obtain

$$
z x_{1}^{i+1} \bar{x}_{2} \bar{x}_{3} x_{1}^{n-i-3} \equiv 0 \quad(\bmod . \operatorname{Id}(\mathcal{V}))
$$

and by putting $x_{3}=x_{1}$ we have

$$
z x_{1}^{i+1} x_{2} x_{1}^{n-i-2} \equiv z x_{1}^{i+2} x_{2} x_{1}^{n-i-3} \quad(\bmod . \operatorname{Id}(\mathcal{V}))
$$

for every $i=0, \ldots, n-3$.

Let $n \geq N$ and consider, for every $j=0, \ldots, n-2$, the polynomials

$$
g_{j}=\bar{x}_{1} x_{1}^{j} \bar{x}_{2} x_{1}^{n-j-2}
$$

corresponding to the standard Young tableaux of shape $\lambda=(n-1,1)$.

Notice that, by the identity (3), we obtain

$$
g_{j} \equiv g_{1} \quad(\bmod . I d(\mathcal{V}))
$$

for $j=2, \ldots, n-2$. It follows that the subspace $\operatorname{span}\left\{g_{0}, \ldots, g_{n-2}\right\}$, modulo $\operatorname{Id}(\mathcal{V})$, has dimension bounded by 2 . Hence $m_{(n-1,1)} \leq 2$.

Our next objective is to find an upper bound for the multiplicity $m_{\lambda}$ for the partition $\lambda=\left(2,1^{n-2}\right)$. 
Proposition 2. Let $\chi_{n}(\mathcal{V})=\sum_{\lambda \vdash n} m_{\lambda} \chi_{\lambda}$. If $n \geq N$ then $m_{\left(2,1^{n-2}\right)} \leq 2$.

Proof. Let $\lambda=\left(3,1^{N-3}\right)$. For every $r=1, \ldots, N-2$ let

$$
f_{r}=\bar{x}_{1} \bar{x}_{2} \cdots \bar{x}_{r} x_{1} \bar{x}_{r+1} \bar{x}_{N-2} x_{1}
$$

be polynomials corresponding to the following standard tableaux

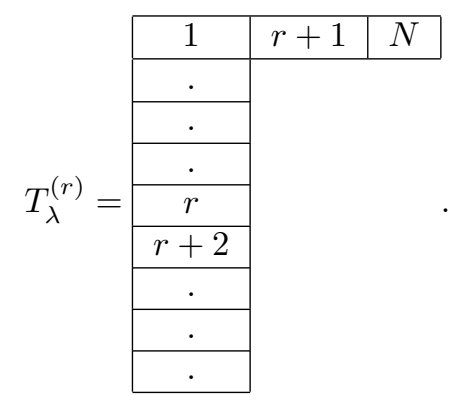

By Lemma 1, it follows that for every $r=1, \cdots, N-2$

$$
\bar{x}_{1} \bar{x}_{2} \cdots \bar{x}_{r} x_{1} \bar{x}_{r+1} \cdots \bar{x}_{N-2} x_{1} \equiv 0 \quad(\bmod . \operatorname{Id}(\mathcal{V})) .
$$

Let consider the substitution $x_{1}=z_{1} z_{2}+x_{1}$ the we obtain

$$
z_{1} z_{2} \bar{x}_{2} \cdots \bar{x}_{r} x_{1} \bar{x}_{r+1} \cdots \bar{x}_{N-2} x_{1} \equiv 0 \quad(\bmod . \operatorname{Id}(\mathcal{V}))
$$

for $r=2, \ldots, N-2$. After multilinearization we have that

$$
z_{1} z_{2} \bar{x}_{2} \cdots \bar{x}_{r} x_{1} \bar{x}_{r+1} \cdots \bar{x}_{N-2} z \equiv-z_{1} z_{2} \bar{x}_{2} \cdots \bar{x}_{r} z \bar{x}_{r+1} \cdots \bar{x}_{N-2} x_{1} \quad(\bmod . \operatorname{Id}(\mathcal{V})) .
$$

If we alternate on $x_{1}, x_{2}, \ldots, x_{N-2}$ it follows that

$$
z_{1} z_{2} \bar{x}_{1} \cdots \bar{x}_{r} \bar{x}_{r+1} \cdots \bar{x}_{N-2} z \equiv \alpha_{r, N} z_{1} z_{2} \bar{x}_{1} \bar{x}_{2} \cdots \bar{x}_{r-1} z \bar{x}_{r} \cdots \bar{x}_{N-2} \quad(\bmod . \operatorname{Id}(\mathcal{V}))
$$

where $r \geq 3$ and $\alpha_{r, N}= \pm 1$ according to the parity of $r$ and $N$.

Let now $n \geq N$, and $f_{1}, \ldots, f_{n-1}$ be polynomials corresponding to the standard Young tableaux of shape $\left(2,1^{n-2}\right)$ in $P_{n}$. Then, if $g_{1}, \ldots, g_{n-1}$ are the polynomials obtained from the $f_{i}$ 's by identifying with $x_{1}$ the two variables of the first row of the corresponding tableaux, for $i=1, \ldots, n-1$, we have that

$$
g_{i}=\bar{x}_{1} \bar{x}_{2} \cdots \bar{x}_{i} x_{1} \bar{x}_{i+1} \cdots \bar{x}_{n-1} .
$$

Let look at the dimension of the $\operatorname{span}\left\{g_{1}, \ldots, g_{n-1}\right\}$.

Notice that, by the identity (4) we obtain

$$
g_{j} \equiv \pm g_{n-1} \quad(\bmod . \operatorname{Id}(\mathcal{V}))
$$

for $j=2, \ldots, n-2$.

It follows that the subspace $\operatorname{span}\left\{g_{1}, \ldots, g_{n-1}\right\}$, modulo $\operatorname{Id}(\mathcal{V})$, has dimension bounded by 2 . Hence $m_{\left(2,1^{n-2}\right)} \leq 2$ and we are done.

Now we are able to prove the following 
Theorem 1. Let $\mathcal{V}$ be a variety of algebras satisfying the identity

$$
x(y z)=0 .
$$

If $c_{n}(\mathcal{V}) \leq C n^{\alpha}$ for some constant $C>0$ and $1<\alpha<2$, then $c_{n}(\mathcal{V}) \leq 4 n+C_{1}$ for some constant $C_{1}>0$.

Proof. Fix $N$ so that, for all $n \geq N, d_{\lambda}>C n^{\alpha}$ for $\lambda \notin\left\{(n),\left(1^{n}\right),(n-1,1),\left(2,1^{n-2}\right)\right\}$. Then by Lemma $1, m_{\lambda}=0$ for every $\lambda \neq(n)(n-1,1),\left(1^{n}\right),\left(2,1^{n-2}\right)$.

Thus for $n \geq N$,

$$
\chi_{n}(\mathcal{V})=m_{(n)} \chi_{(n)}+m_{(n-1,1)} \chi_{(n-1,1)}+m_{\left(1^{n}\right)} \chi_{\left(1^{n}\right)}+m_{\left(2,1^{n-2}\right)} \chi_{\left(2,1^{n-2}\right)} .
$$

Since $\operatorname{deg} \chi_{(n-1,1)}=\operatorname{deg} \chi_{\left(2,1^{n-2}\right)}=n-1$ and $\operatorname{deg} \chi_{\left(1^{n}\right)}=\operatorname{deg} \chi_{\left(1^{n}\right)}=1$, by recalling Remark 1, Proposition 1 and Proposition 2, we get

$$
c_{n}(\mathcal{V}) \leq 1+2(n-1)+1+2(n-1) \leq 4 n-2 .
$$

For $1 \leq n<N$, let $C_{1}$ be such that $c_{n}(\mathcal{V}) \leq 4 n+C_{1}$ and we are done.

\section{Varieties $\mathcal{V}$ such that $c_{n}(\mathcal{V}) \leq C n^{\alpha}, 2<\alpha<3$}

Let $\mathcal{V}$ be the variety of algebras satisfying the identity

$$
x(y z) \equiv 0 .
$$

Throughout this section we shall assume that $c_{n}(\mathcal{V}) \leq C n^{\alpha}$, for some constants $C$ and $\alpha, 2<\alpha<3$.

Our aim is to prove that for such variety $c_{n}(\mathcal{V})<C_{1} n^{2}$, for some constant $C_{1}$.

Let observe that if $\lambda \in\left\{(n),\left(1^{n}\right),(n-1,1),\left(2,1^{n-2}\right)\right\}$, then $m_{\lambda} d_{\lambda}<n^{2}$, so from now on we shall consider partitions $\lambda \notin\left\{(n),\left(1^{n}\right),(n-1,1),\left(2,1^{n-2}\right)\right\}$. The strategy of the proof will be the following: we shall first prove that, for $n$ large enough, $m_{\lambda}=0$ for every $\lambda \notin\left\{(n-2,1,1),\left(3,1^{n-3}\right),(n-2,2),\left(2,2,1^{n-4}\right)\right\}$ then for the above case we shall find an upper bound for the multiplicities $m_{\lambda}$.

Let start with the following

Lemma 2. If $\lambda \vdash n, n \neq 6$, is such that $\lambda \notin\left\{(n-2,1,1),\left(3,1^{n-3}\right),(n-2,2),\left(2,2,1^{n-4}\right)\right\}$ then $d_{\lambda} \geq \frac{n^{3}}{44}$.

Proof. Let $\lambda=\left(\lambda_{1}, \lambda_{2}, \ldots\right) \vdash n$ and let denote by $\lambda^{\prime}=\left(\lambda_{1}^{\prime}, \ldots, \lambda_{s}^{\prime}\right) \vdash n$ the conjugate partition of $\lambda$. It easy to check that for any $n \neq 6$

$$
d_{(n-3,2,1)}=d_{\left(3,2,1^{n-5}\right)}>d_{(n-3,1,1,1)}=d_{\left(3,2,1^{n-5}\right)}>d_{(n-3,3)}=d_{\left(2,2,2,1^{n-6}\right)}>\frac{n^{3}}{44} .
$$

It follows that if $\lambda_{1}=n-3$, or $\lambda_{1}^{\prime}=n-3$ or $n \leq 9$ then the conclusion of the lemma follows by direct computation from the hook formula (see [8]).

Hence we may assume that $n \geq 10, \lambda_{1} \leq n-4$ and $\lambda_{1}^{\prime}<n-4$. The proof will be by induction on $n$. If the shape of the diagram of $\lambda$ is not a rectangle then there exist two subdiagrams corresponding to partitions $\mu$ and $\nu$ each containing $n-1$ boxes and satisfying 
the hypotheses of the lemma. But then, by using induction and the branching rule, we have that

$$
d_{\lambda} \geq d_{\mu}+d_{\nu} \geq 2 \cdot \frac{(n-1)^{3}}{44}>\frac{n^{3}}{44} .
$$

If the shape of the diagram of $\lambda$ is a rectangle, there exist two different subdiagrams corresponding to partitions $\mu$ and $\nu$, each with $n-2$ boxes, and they both satisfy the hypotheses of the lemma. In this case we have

$$
d_{\lambda} \geq d_{\mu}+d_{\nu} \geq 2 \cdot \frac{(n-2)^{3}}{44}>\frac{n^{3}}{44} .
$$

In fact let consider the sequence

$$
a_{n}=2 \cdot \frac{(n-2)^{3}}{44}-\frac{n^{3}}{44}=\frac{(\sqrt[3]{2}(n-2)-n)\left((\sqrt[3]{2}(n-2))^{2}+\sqrt[3]{2}(n-2) n+n^{2}\right)}{44} .
$$

As $n \geq 10>\frac{2 \sqrt[3]{2}}{\sqrt[3]{2}-1}$ then $\sqrt[3]{2}(n-2)-n>0$. So, $a_{n}>0$ for any $n \geq 10$ and we are done.

From the above lemma it follows that if $\lambda \vdash n$ is distinct from $(n-2,1,1),\left(3,1^{n-3}\right),(n-$ $2,2),\left(2,2,1^{n-4}\right)$, then there exists $m>0$ such that for all $n \geq m$ we have that $d_{\lambda}>\frac{n^{3}}{44}>$ $C n^{\alpha}$ and so $m_{\lambda}=0$. We fix the integer $m \geq 4$ from now and we shall also assume that the integer $m$ has the further property that $c_{n}(\mathcal{V})<\frac{n^{3}}{44}$ for all $n \geq m$.

Let start with the following

Lemma 3. There exists $r$, with $0 \leq r \leq m-3$, such that

$$
z_{1} z_{2} x_{1}^{r} z x_{1}^{m-r-2} \equiv \sum_{i>r} \gamma_{i} z_{1} z_{2} x_{1}^{i} z x_{1}^{m-i-2} \quad(\bmod . \operatorname{Id}(\mathcal{V}))
$$

where, for some $i, \gamma_{i} \neq 0$.

Proof. Let $\lambda=(m-2,1,1) \vdash m$. For $p=0, \ldots, m-3$, we define the tableaux

$$
T_{\lambda}^{(p)}=\begin{array}{|c|c|c|c|c|c|}
\hline 1 & 2 & \cdots & p+1 & p+4 & \cdots \\
\hline p+2 & \multicolumn{7}{|l}{} \\
\cline { 1 - 1 } p+3 &
\end{array}
$$

and we associate to $T_{\lambda}^{(p)}$ the left-normed polynomials

$$
g_{p}=\bar{x}_{1} x_{1}^{p} \bar{x}_{2} \bar{x}_{3} x_{1}^{m-p-3} .
$$

Notice that, for every $p=0, \ldots, m-3$, the polynomials $g_{p}$ are obtained from the essential idempotents corresponding to the tableaux $T_{\lambda}^{(p)}$ by identifying all the elements in each row of $\lambda$.

If the polynomials $g_{p}$ are linearly independent then $m_{\lambda} \geq m-2$. In this case, by Lemma $1, d_{\lambda}>\frac{m^{2}}{8}$ and we have

$$
c_{m}(\mathcal{V}) \geq(m-2) \frac{m^{2}}{8}>\frac{m^{3}}{44}>C m^{\alpha}
$$

a contradiction. So, $m_{\lambda}$ will be less than $m-2$ then it follows that the polynomials $g_{p}$ are linearly dependent and this implies 


$$
\sum_{p=0}^{m-3} \alpha_{p} \bar{x}_{1} x_{1}^{p} \bar{x}_{2} \bar{x}_{3} x_{1}^{m-p-3} \equiv 0 \quad(\bmod . \operatorname{Id}(\mathcal{V})) .
$$

Let us replace $x_{3}$ with $z_{1} z_{2}$ and $x_{2}$ with $z$ then we obtain

$$
\sum_{p=0}^{m-3} \alpha_{p} z_{1} z_{2} x_{1}^{p}\left(z x_{1}-x_{1} z\right) x_{1}^{m-p-3} \equiv 0 \quad(\bmod . \operatorname{Id}(\mathcal{V})) .
$$

Let now $r$ be the minimum $p$ such that $\alpha_{p} \neq 0$ and $t$ be the maximum $p$ such that $\alpha_{p} \neq 0$. If $t=r$ then

$$
z_{1} z_{2} x_{1}^{r} z x_{1}^{m-r-2} \equiv z_{1} z_{2} x_{1}^{r+1} z x_{1}^{m-r-1} \quad(\bmod . \operatorname{Id}(\mathcal{V}))
$$

If $t \neq r$ then

$$
\sum_{i=r}^{t+1} \beta_{i} z_{1} z_{2} x_{1}^{i} z x_{1}^{m-i-2} \equiv 0 \quad(\bmod . \operatorname{Id}(\mathcal{V}))
$$

where $\beta_{r}=\alpha_{r} \neq 0, \beta_{t+1}=-\alpha_{t} \neq 0$ and $\beta_{r+j}=\alpha_{r+j}-\alpha_{r+j-1}$ for all $1 \leq j<t-r+1$. It follows that

$$
z_{1} z_{2} x_{1}^{r} z x_{1}^{m-r-2} \equiv \sum_{i=r+1}^{t+1} \frac{\beta_{i}}{\beta_{r}} z_{1} z_{2} x_{1}^{i} z x_{1}^{m-i-2}=\sum_{i=r+1}^{t+1} \gamma_{i} z_{1} z_{2} x_{1}^{i} z x_{1}^{m-i-2} \quad(\bmod . \operatorname{Id}(\mathcal{V}))
$$

where $\gamma_{t+1}=-\frac{\beta_{t+1}}{\beta_{r}}=\frac{\alpha_{t}}{\alpha_{r}} \neq 0$.

From this lemma it easily follows the following

Remark 2. If $s \geq m$ and $t \geq m$ then

$$
z_{1} x_{1}^{s} z x_{1}^{t} \equiv \sum_{i} \gamma_{i} z_{1} x_{1}^{s_{i}} z x_{1}^{t_{i}} \quad(\bmod . \operatorname{Id}(\mathcal{V}))
$$

where $t_{i}<m$.

Lemma 4. Let $k=2 m$. For a fixed $q$, with $0 \leq q<m$, there exists $r_{q}, 0 \leq r_{q} \leq k-q-3$, such that

$$
\left.z_{1} z_{2} x_{1}^{r_{q}} \bar{x}_{2} x_{1}^{q} \bar{x}_{3} z x_{1}^{k-r_{q}-q-3} \equiv \sum_{0 \leq i<r_{q}} \beta_{i} z_{1} z_{2} x_{1}^{i} \bar{x}_{2} x_{1}^{q} \bar{x}_{3} z x_{1}^{k-i-q-3} \quad \text { (mod. } \operatorname{Id}(\mathcal{V})\right) .
$$

Proof. Let $k=2 m$. For a fixed $q, 0 \leq q<m$, and for $p=0, \ldots, k-q-3$ let

$$
h_{p, q}=\bar{x}_{1} x_{1}^{p} \bar{x}_{2} x_{1}^{q} \bar{x}_{3} x_{1}^{k-p-q-3}
$$

be the left normed polynomials associated to the tableaux

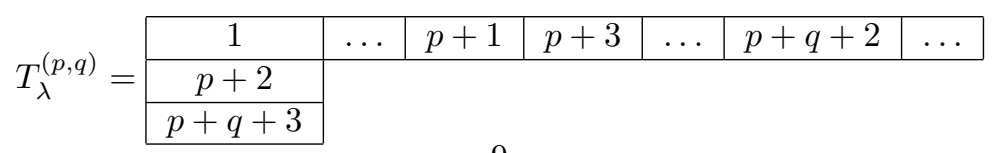


where $\lambda=(k-2,1,1) \vdash k$.

As in the previous lemma, since $m \geq 4$, if $m_{\lambda} \geq k-q-2$ then,

$$
(k-q-2) \frac{(2 m)^{2}}{8}>(k-m-2) \frac{(2 m)^{2}}{8}=(m-2) \frac{m^{2}}{2}>\frac{(2 m)^{3}}{44}>C k^{\alpha} \geq c_{k}(\mathcal{V})
$$

a contradiction.

Then the polynomials $h_{p, q}$, for any $q$, must be linearly dependent and so

$$
\sum_{p=0}^{k-q-3} \alpha_{p} h_{p, q} \equiv 0 \quad(\bmod . \operatorname{Id}(\mathcal{V})) .
$$

Let now consider the following substitution $x_{1}=z_{1} z_{2}+x_{1}$ and let $r_{q}$ be the maximum $p$ such that $\alpha_{p} \neq 0$. It follows that

$$
z_{1} z_{2} x_{1}^{r_{q}} \bar{x}_{2} x_{1}^{q} \bar{x}_{3} x_{1}^{k-r_{q}-q-3} \equiv \sum_{0 \leq i<r_{q}} \beta_{i} z_{1} z_{2} x_{1}^{i} \bar{x}_{2} x_{1}^{q} \bar{x}_{3} x_{1}^{k-i-q-3} \quad(\bmod . \operatorname{Id}(\mathcal{V})),
$$

and we are done.

From now on we shall assume that $k=2 \mathrm{~m}$.

We have the following

Remark 3. For a fixed $q, 0 \leq q<m$, and $s, t \geq k$ then either

$$
z_{1} x_{1}^{s} \bar{x}_{2} x_{1}^{q} \bar{x}_{3} x_{1}^{t} \equiv \sum_{i<k} \beta_{i} z_{1} x_{1}^{i} \bar{x}_{2} x_{1}^{q} \bar{x}_{3} x_{1}^{s+t-i} \quad(\bmod . \operatorname{Id}(\mathcal{V}))
$$

where $\beta_{i} \neq 0$ for some $i$, or

$$
z_{1} x_{1}^{s} \bar{x}_{2} x_{1}^{q} \bar{x}_{3} x_{1}^{t} \equiv 0 \quad(\bmod . \operatorname{Id}(\mathcal{V}))
$$

Proof. Let consider the polynomial $z_{1} x_{1}^{s} \bar{x}_{2} x_{1}^{q} \bar{x}_{3} x_{1}^{t}$ with $q<m, s \geq k$ and $t \geq k$.

If in (6) there exists $i$ such that $\beta_{i} \neq 0$, then

$$
z_{1} x_{1}^{s} \bar{x}_{2} x_{1}^{q} \bar{x}_{3} x_{1}^{t} \equiv \sum_{i<k} \beta_{i} z_{1} x_{1}^{i} \bar{x}_{2} x_{1}^{q} \bar{x}_{3} x_{1}^{s+t-i} \quad(\bmod . \operatorname{Id}(\mathcal{V}))
$$

and we are done.

Otherwise if in (6), $\beta_{i}=0$ for all $i$, then we have

$$
z_{1} z_{2} x_{1}^{r_{q}} \bar{x}_{2} x_{1}^{q} \bar{x}_{3} x_{1}^{k-r_{q}-q-3} \equiv 0 \quad(\bmod . \operatorname{Id}(\mathcal{V})) .
$$

So, for any $s, t \geq k$, and $0 \leq q<m$ it follows that

$$
z_{1} x_{1}^{s} \bar{x}_{2} x_{1}^{q} \bar{x}_{3} x_{1}^{t} \equiv 0 \quad(\bmod . \operatorname{Id}(\mathcal{V})) .
$$

Proposition 3. Let $\chi_{n}(\mathcal{V})=\sum_{\lambda \vdash n} m_{\lambda} \chi_{\lambda}$. If $\lambda=(n-2,1,1)$ then $m_{(n-2,1,1)} \leq 5 m^{2}$. 
Proof. If $n<k$ then $m_{\lambda} \leq d_{\lambda}<n^{2}<5 m^{2}$.

Let $n \geq k$. The highest weight vectors corresponding to the standard Young tableaux of shape $\lambda=(n-2,1,1)$ are of the following type

$$
g_{\alpha, \beta, \gamma}=\bar{x}_{1} x_{i}^{\alpha} \bar{x}_{2} x_{1}^{\beta} \bar{x}_{3} x_{1}^{\gamma}
$$

where $\alpha+\beta+\gamma=n-3$. We want to find an upper bound for the dimension of the span $\left\{g_{\alpha, \beta, \gamma}\right\}$.

Let first assume $0 \leq \beta<m$.

If $\alpha \geq k$ and $\gamma \geq k$, by Remark 3, either $g_{\alpha, \beta, \gamma} \equiv 0$ or we can write $g_{\alpha, \beta, \gamma}$ as a linear combination of polynomials $\bar{x}_{1} x_{i}^{\alpha^{\prime}} \bar{x}_{2} x_{1}^{\beta} \bar{x}_{3} x_{1}^{\gamma^{\prime}}$ where $\alpha^{\prime}<k$.

Hence, if $\beta<m$ we have to consider polynomials $g_{\alpha, \beta, \gamma}$ such that either $\alpha<k$ or $\gamma<k$ and so we obtain at most $2 \mathrm{~km}$ polynomials.

Let now $\beta \geq m$.

If $\gamma \geq m$ then, by Remark 2, we can write $g_{\alpha, \beta, \gamma}$ as a linear combination of polynomials $g_{\alpha^{\prime}, \beta^{\prime}, \gamma^{\prime}}$ where $\gamma^{\prime}<m$.

So let consider polynomials $g_{\alpha, \beta, \gamma}$ with $\beta \geq m$ and $\gamma<m$.

If $\alpha \geq m$ then, by Remark $2, g_{\alpha, \beta, \gamma}$ is equivalent to a linear combination of polynomials $g_{\alpha^{\prime}, \beta^{\prime}, \gamma^{\prime}}$ where $\beta^{\prime}<m$.

So we have to consider polynomials $g_{\alpha, \beta, \gamma}$ with $\alpha<m, \beta \geq m$ and $\gamma<m$ and this are at most $m^{2}$

It follows that the $\operatorname{dim} \operatorname{span}\left\{g_{\alpha, \beta, \gamma}\right\} \leq 5 m^{2}$.

\section{Lemma 5 .}

1) There exists $r, 0 \leq r \leq m-2$, such that

$$
z_{1} z_{2} \bar{x}_{1} \cdots \bar{x}_{r} z \bar{x}_{r+1} \cdots \bar{x}_{m-2} \equiv \sum_{i>r} \gamma_{i} z_{1} z_{2} \bar{x}_{1} \cdots \bar{x}_{i} z \bar{x}_{i+1} \cdots \bar{x}_{m-2} \quad(\bmod . \operatorname{Id}(\mathcal{V})),
$$

where $\gamma_{r+1}=-1$.

2) Let $k=2 m$, then for any fixed $q, 0 \leq q<m$, there exists $r_{q}, 0<r_{q}<k-q-2$, such that

$$
\begin{gathered}
z_{1} z_{2} \bar{x}_{2} \cdots \bar{x}_{r_{q}} z \bar{x}_{r_{q}+1} \cdots \bar{x}_{r_{q}+q} z \bar{x}_{r_{q}+q+1} \cdots \bar{x}_{k-2} \equiv \\
\sum_{i<r_{q}} \beta_{i} z_{1} z_{2} \bar{x}_{2} \cdots \bar{x}_{i} z \bar{x}_{i+1} \cdots \bar{x}_{i+q} z \bar{x}_{i+q+1} \cdots \bar{x}_{k-2} \quad(\bmod . \operatorname{Id}(\mathcal{V}))
\end{gathered}
$$

Proof. 1) Let $\lambda=\left(3,1^{m-3}\right) \vdash m$. For every $p \in P=\{1,3,5, \cdots\}$, we define the standard tableaux

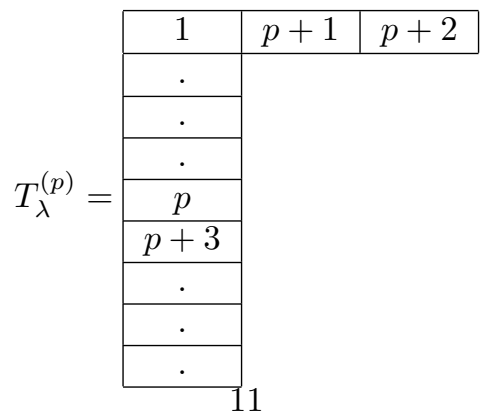


and we associate to $T_{\lambda}^{(p)}$ the left-normed polynomials

$$
g_{p}=\bar{x}_{1} \cdots \bar{x}_{p} x_{1} x_{1} \bar{x}_{p+1} \cdots \bar{x}_{m-2} .
$$

Notice that for every $p \in P=\{1,3,5, \cdots\}$, the $[(m+1) / 2]$ polynomials $g_{p}$ are obtained from the essential idempotents corresponding to the tableaux $T_{\lambda}^{(p)}$ by identifying all the elements in each row of $\lambda$. Since $c_{m}(\mathcal{V})<\frac{m^{3}}{44}<[(m+1) / 2] \frac{m^{2}}{8}$ it follows that the polynomials $g_{p}$ are linearly dependent then

$$
\sum_{p \in P} \alpha_{p} g_{p} \equiv 0 \quad(\bmod . \operatorname{Id}(\mathcal{V}))
$$

By making the substitution $x_{1}=z_{1} z_{2}+x_{1}$ we obtain

$$
\sum_{p \in P} \alpha_{p} z_{1} z_{2} \bar{x}_{2} \cdots \bar{x}_{p} x_{1} x_{1} \bar{x}_{p+1} \cdots \bar{x}_{m-2} \equiv 0 \quad(\bmod . \operatorname{Id}(\mathcal{V})) .
$$

Let $r$ be the minimum $p$ such that $\alpha_{p} \neq 0$, then

$$
z_{1} z_{2} \bar{x}_{2} \cdots \bar{x}_{r} x_{1} x_{1} \bar{x}_{r+1} \cdots \bar{x}_{m-2} \equiv \sum_{i>r} \beta_{i} z_{1} z_{2} \bar{x}_{2} \cdots \bar{x}_{i} x_{1} x_{1} \bar{x}_{i+1} \cdots \bar{x}_{m-2} \quad(\bmod \operatorname{Id}(\mathcal{V})) \text {. }
$$

By substituting $x_{1}$ with $x_{1}+z_{1}$ and by alternating on $x_{1}, x_{2}, \ldots, x_{m-2}$ we obtain

$$
z_{1} z_{2} \bar{x}_{1} \cdots \bar{x}_{r} z \bar{x}_{r+1} \cdots \bar{x}_{m-2} \equiv \sum_{i>r} \gamma_{i} z_{1} z_{2} \bar{x}_{1} \cdots \bar{x}_{i} z \bar{x}_{i+1} \cdots \bar{x}_{N-2} \quad(\bmod . \operatorname{Id}(\mathcal{V})) .
$$

Let observe that $\gamma_{r+1}=-1$ and we are done.

2) Let now $k=2 m$, for a fixed $q=0, \ldots, m-1$ and for $p=1,2, \ldots, k-q-2$ let

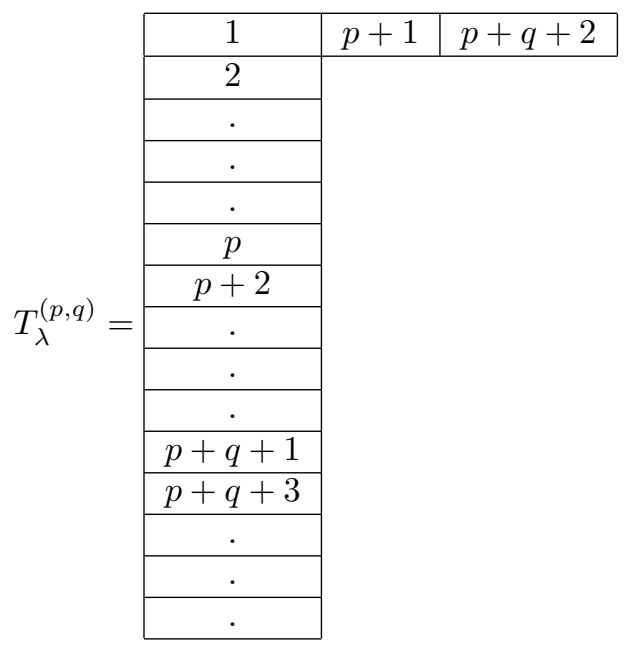

be standard tableaux of shape $\lambda=\left(3,1^{k-3}\right) \vdash k$. We associate to any tableaux $T_{\lambda}^{(p, q)}, p=$ $1,2, \ldots, k-q-2$, the left-normed polynomials

$$
h_{p, q}=\bar{x}_{1} \cdots \bar{x}_{p} x_{1} \bar{x}_{p+1} \cdots \bar{x}_{p+q} x_{1} \bar{x}_{p+q+1} \cdots \bar{x}_{k-2} .
$$


As before, by the hypothesis on $c_{k}(\mathcal{V})$ we have that the polynomials $h_{p, q}$ are linearly dependent then

$$
\sum \alpha_{p, q} h_{p, q} \equiv 0 \quad(\bmod . \operatorname{Id}(\mathcal{V}))
$$

Let $r_{q}>0$ be the maximum $p$ such that $\alpha_{p, q} \neq 0$, then after the substitution $x_{1}=z_{1} z_{2}+z$ we obtain

$$
\begin{gathered}
z_{1} z_{2} \bar{x}_{2} \cdots \bar{x}_{r_{q}} z \bar{x}_{r_{q}+1} \cdots \bar{x}_{r_{q}+q} z \bar{x}_{r_{q}+q+1} \cdots \bar{x}_{k-2} \equiv \\
\sum_{i<r_{q}} \beta_{i} z_{1} z_{2} \bar{x}_{2} \cdots \bar{x}_{i} z \bar{x}_{i+1} \cdots \bar{x}_{i+q} z \bar{x}_{i+q+1} \cdots \bar{x}_{k-2} \quad(\bmod . \operatorname{Id}(\mathcal{V}))
\end{gathered}
$$

and we are done.

\section{Remark 4.}

1) If $s \geq m$ and $t \geq m$ then

$$
z_{1} \bar{x}_{1} \cdots \bar{x}_{s} z \bar{x}_{s+1} \cdots \bar{x}_{s+t} \equiv \sum_{i} \gamma_{i} z_{1} \bar{x}_{1} \cdots \bar{x}_{i} z \bar{x}_{i+1} \cdots \bar{x}_{i+t^{\prime}} \quad(\bmod . \operatorname{Id}(\mathcal{V})),
$$

where $t^{\prime}<m$.

2) If $0 \leq q<m, s \geq k$ and $t \geq k$ then either

$$
\begin{gathered}
\bar{x}_{1} \cdots \bar{x}_{s} z \bar{x}_{s+1} \cdots \bar{x}_{s+q} z \bar{x}_{s+q+1} \cdots \bar{x}_{s+q+t} \equiv \\
\sum_{s^{\prime}<k} \gamma_{s^{\prime}} \bar{x}_{1} \cdots \bar{x}_{s^{\prime}} z \bar{x}_{s^{\prime}+1} \cdots \bar{x}_{s^{\prime}+q} z \bar{x}_{s^{\prime}+q+1} \cdots \bar{x}_{s^{\prime}+q+t^{\prime}} \quad(\bmod . \operatorname{Id}(\mathcal{V}))
\end{gathered}
$$

where $\gamma_{s^{\prime}} \neq 0$, for some $s^{\prime}$ or

$$
z_{1} \bar{x}_{1} \cdots \bar{x}_{s} z \bar{x}_{s+1} \cdots \bar{x}_{s+t} \equiv 0 \quad(\bmod . \operatorname{Id}(\mathcal{V})) .
$$

Proof. 1) Let consider the polynomial $z_{1} \bar{x}_{1} \cdots \bar{x}_{s} z \bar{x}_{s+1} \cdots \bar{x}_{s+t}$. If $s \geq m$ and $t \geq m$, by (7) it follows that

$$
z_{1} \bar{x}_{1} \cdots \bar{x}_{s} z \bar{x}_{s+1} \cdots \bar{x}_{s+t} \equiv \sum_{i} \gamma_{i} z_{1} \bar{x}_{1} \cdots \bar{x}_{i} z \bar{x}_{i+1} \cdots \bar{x}_{i+t^{\prime}} \quad(\bmod . \operatorname{Id}(\mathcal{V})),
$$

where $t^{\prime}<m$.

2) Let now consider the polynomial $\bar{x}_{1} \cdots \bar{x}_{s} z \bar{x}_{s+1} \cdots \bar{x}_{s+q} z \bar{x}_{s+q+1} \cdots \bar{x}_{s+q+t}$ with $0 \leq$ $q<m, s \geq k$ and $t \geq k$. If in (8), $\beta_{i} \neq 0$ for some $i$, then

$$
\begin{gathered}
\bar{x}_{1} \cdots \bar{x}_{s} z \bar{x}_{s+1} \cdots \bar{x}_{s+q} z \bar{x}_{s+q+1} \cdots \bar{x}_{s+q+t} \equiv \\
\sum_{s^{\prime}<k} \gamma_{s^{\prime}} \bar{x}_{1} \cdots \bar{x}_{s^{\prime}} z \bar{x}_{s^{\prime}+1} \cdots \bar{x}_{s^{\prime}+q} z \bar{x}_{s^{\prime}+q+1} \cdots \bar{x}_{s^{\prime}+q+t^{\prime}} \quad(\bmod . \operatorname{Id}(\mathcal{V})),
\end{gathered}
$$

where $\gamma_{s^{\prime}} \neq 0$, for some $s^{\prime}$.

If in (8), $\beta_{i}=0$ for any $i$, then we have

$$
z_{1} z_{2} \bar{x}_{2} \cdots \bar{x}_{r_{q}} z \bar{x}_{r_{q}+1} \cdots \bar{x}_{r_{q}+q} z \bar{x}_{r_{q}+q+1} \cdots \bar{x}_{k-2} \equiv 0 \quad(\bmod . \operatorname{Id}(\mathcal{V}))
$$

and this implies that

$$
\bar{x}_{1} \cdots \bar{x}_{s} z \bar{x}_{s+1} \cdots \bar{x}_{s+q} z \bar{x}_{s+q+1} \cdots \bar{x}_{s+q+t} \equiv 0 \quad(\bmod . \operatorname{Id}(\mathcal{V})),
$$

for $0 \leq q<m$ and $s, t \geq k$. 
Proposition 4. Let $\lambda=\left(3,1^{n-3}\right) \vdash n$, then $m_{\lambda} \leq 5 m^{2}$.

Proof. If $n<k$ then $m_{\lambda} \leq d_{\lambda}<n^{2}<5 m^{2}$.

So, let $n \geq k$. For $p=1, \cdots n-2$ and $p+q+r=n-2$ let

$$
f_{p, q, r}=\underbrace{\bar{x}_{1} \bar{x}_{2} \cdots \bar{x}_{p}}_{p} x_{1} \underbrace{\bar{x}_{p+1} \cdots \bar{x}_{p+q}}_{q} x_{1} \underbrace{\bar{x}_{p+q+1} \cdots \bar{x}_{n-2}}_{r}
$$

be the highest weight vectors corresponding to the standard tableaux of the partition $\left(3,1^{n-3}\right)$. The dimension of the space spanned by all $f_{p, q, r}$, equals $m_{\lambda}$. We want to find an upper bound of this dimension.

Let first assume that $q<m$.

If $p>k$ and $r>k$, by Remark $4, f_{p, q, r}$ is equivalent to a linear combination of polynomials of the type $f_{p^{\prime}, q, r^{\prime}}$ where $p^{\prime}<k$, and these polynomials are at most $k m$.

If $p>k$ and $r<k$ we obtain again at most $k m$ polynomials.

So let assume that $q \geq m$.

If $p>m$, by the first part of Remark 4 , we obtain that $f_{p, q, s}$ is a linear combination of polynomials $f_{p^{\prime}, q^{\prime}, r^{\prime}}$ with $q^{\prime}<m$.

So let suppose $p<m, q \geq m$, and $r>m$. By Remark 4, we can write $f_{p, q, r}$ as a linear combination of polynomials $f_{p^{\prime}, q^{\prime}, r^{\prime}}$ with $r^{\prime}<m$. It follows that, if $q \geq m$, the linearly independent polynomials $f_{p, q, r}$ are at most $m^{2}$.

As consequence we obtain that $m_{\lambda} \leq 5 \mathrm{~m}^{2}$.

Next we shall prove that for $\lambda=(n-2,2) \vdash n$ the multiplicity $m_{\lambda}$ is bounded by some constant.

Lemma 6. For any $\alpha, \beta, \gamma, \delta, \eta$ with $\alpha+\beta+\gamma+\delta+\eta \geq m-5$ we have that

$$
\begin{aligned}
& z_{1} z_{2} x_{1}^{\alpha} \bar{y}_{1} x_{1}^{\beta} \bar{y}_{2} x_{1}^{\gamma} \tilde{y}_{1} x_{1}^{\delta} \tilde{y}_{2} x_{1}^{\eta} \equiv 0 \quad(\bmod . \operatorname{Id}(\mathcal{V})), \\
& z_{1} z_{2} x_{1}^{\alpha} \bar{y}_{1} x_{1}^{\beta} \tilde{y}_{1} x_{1}^{\gamma} \tilde{y}_{2} x_{1}^{\delta} \bar{y}_{2} x_{1}^{\eta} \equiv 0 \quad(\bmod . \operatorname{Id}(\mathcal{V})) .
\end{aligned}
$$

Proof. Let $\lambda=(2,2,1) \vdash 5$ and $\mu=(n-5) \vdash n-5$. Let $M_{\lambda} \hat{\otimes} M_{\mu}$ be the the $S_{n^{-}}$module outer tensor product of the irreducible modules $M_{\lambda}$ and $M_{\mu}$, (see [7]). If we consider the polynomials

$$
\begin{aligned}
& \bar{y}_{3} x_{1}^{\alpha} \bar{y}_{1} x_{1}^{\beta} \bar{y}_{2} x_{1}^{\gamma} \tilde{y}_{1} x_{1}^{\delta} \tilde{y}_{2} x_{1}^{\eta} \\
& \bar{y}_{3} x_{1}^{\alpha} \bar{y}_{1} x_{1}^{\beta} \tilde{y}_{1} x_{1}^{\gamma} \tilde{y}_{2} x_{1}^{\delta} \bar{y}_{2} x_{1}^{\eta}
\end{aligned}
$$

obtained from the two modules $M_{\lambda}$ and $M_{\mu}$, from remark after Lemma 2, it follows that these polynomials are identities of the variety $\mathcal{V}$. Let substitute $y_{3}$ with $z_{1} z_{2}$ and we are done.

Proposition 5. Let $\lambda=(n-2,2) \vdash n$ then $m_{\lambda} \leq \bar{C}$ where $\bar{C}=\max \left\{4 m+3, m^{2}\right\}$.

Proof. We shall construct polynomials corresponding to essential idempotents of the group algebra of $S_{n}$. Let $e_{T_{\lambda}} \in F S_{n}$ be the essential idempotent corresponding to the tableau $T_{\lambda}$, we shall identify $e_{T_{\lambda}}$ with the polynomial $e_{T_{\lambda}}\left(x_{n}, \ldots, x_{1}\right)=e_{T_{\lambda}} x_{n} \cdots x_{1}$ obtained by acting with $e_{T_{\lambda}}$ on the left normed monomial $x_{n} \cdots x_{1}$. We shall then identify all variables corresponding to each row of the tableau. Let consider the standard Young tableaux corresponding to $\lambda=(n-2,2)$. This tableaux are of different types. 
Type 1. Let consider first tableaux of the following types

$$
T_{\lambda}^{i}=\begin{array}{|l|l|l|l|l|l|}
\hline 1 & 2 & 3 & \cdots & n-1 & n \\
\hline i & j & \multicolumn{4}{|c|}{} \\
\hline
\end{array}
$$

where $4 \leq i<j \leq n-2$ or

$$
T_{\lambda}^{i}=\begin{array}{|l|l|l|l|l|}
\hline 1 & 2 & \cdots & n-1 & n \\
\hline 3 & i & \multicolumn{4}{|c}{} \\
\hline
\end{array}
$$

where $4 \leq i \leq n-2$ or

$$
T_{\lambda}^{i}=\begin{array}{|l|l|l|l|l|}
\hline 1 & 3 & \cdots & n-1 & n \\
\hline 2 & i & \multicolumn{4}{|c}{} \\
\hline
\end{array}
$$

where $4 \leq i \leq n-2$.

For this tableaux we obtain, by Lemma 6 , that $e_{T_{\lambda}^{i}} \equiv 0$.

Type 2. Let now consider tableaux of the following type

$$
T_{\lambda}^{i}=\begin{array}{|l|l|l|l|l|}
\hline 1 & 3 & \cdots & n-2 & n-1 \\
\hline 2 & n & \multicolumn{3}{|l}{} \\
\hline
\end{array}
$$

or

$$
T_{\lambda}^{i}=\begin{array}{|c|c|c|c|c|}
\hline 1 & 3 & \cdots & n-2 & n \\
\hline 2 & n-1 & \multicolumn{3}{|c}{} \\
\hline
\end{array}
$$

In the first case we obtain

$$
e_{T_{\lambda}^{i}}=\tilde{x}_{2} x_{1}^{n-4} \tilde{x}_{1} \bar{x}_{2} \bar{x}_{1}
$$

and in the second case

$$
e_{T_{\lambda}^{i}}=x_{1} \tilde{x}_{2} x_{1}^{n-3} \tilde{x}_{1} \bar{x}_{2} \bar{x}_{1} .
$$

Type 3. If

$$
T_{\lambda}^{i}=\begin{array}{|c|c|c|c|}
\hline 1 & 2 & \cdots & n-2 \\
\hline n-1 & n & \multicolumn{2}{|c}{} \\
\hline
\end{array}
$$

then we obtain the polynomial

$$
e_{T_{\lambda}^{i}}=\tilde{x}_{2} \bar{x}_{2} x_{1}^{n-4} \tilde{x}_{1} \bar{x}_{1}
$$

Type 4. Let now

$$
T_{\lambda}^{i}=\begin{array}{|c|c|c|c|}
\hline 1 & 2 & \cdots & n \\
\hline i & n-1 & \multicolumn{2}{|c}{} \\
\hline
\end{array}
$$

where $2<i<n-1$ then $e_{T_{\lambda}^{i}}$ are polynomials of the following type

$$
x_{1} \tilde{x}_{2} x_{1}^{n-i-2} \bar{x}_{2} x_{1}^{i-3} \tilde{x}_{1} \bar{x}_{1} .
$$

By Lemma 3 and Remark 2, it follows that they are linear combination of polynomials of the same type with $n-i-2<m$ or $i-3<m$. So, in this case the linearly independent polynomials are less than $2 m$. 
Type 5. Let now consider the tableaux

$$
T_{\lambda}^{i}=\begin{array}{|l|l|l|l|}
\hline 1 & 2 & \cdots & n-1 \\
\hline i & n & \multicolumn{2}{|l}{} \\
\hline
\end{array}
$$

where $2<i<n-1$ then we obtain the polynomials

$$
e_{T_{\lambda}^{i}}=\tilde{x}_{2} x_{1}^{n-i-1} \bar{x}_{2} x_{1}^{i-3} \tilde{x}_{1} \bar{x}_{1}
$$

By Remark 2, these polynomials are linear combinations of polynomials of the same type with $n-i-2<m$ or $i-3<m$. So, also in this case the polynomials that are linearly independent are less than $2 m$.

It follows that, for $n \geq m, m_{\lambda} \leq 4 m+3$.

If $n<m$ then $m_{\lambda} \leq \bar{d}_{\lambda}<m^{2}$. Hence for any $n, m_{\lambda} \leq \bar{C}$ where $\bar{C}=\max \left\{4 m+3, m^{2}\right\}$.

Now we shall prove that the multiplicity for the partition $\lambda=\left(2,2,1^{n-4}\right) \vdash n$ is bounded by some constant.

Lemma 7. For any $\alpha, \beta, \gamma, \delta, \eta$ with $\alpha+\beta+\gamma+\delta+\eta \geq m-5$ we have

$$
\begin{gathered}
z_{1} z_{2} \bar{x}_{1} \bar{x}_{2} \ldots \bar{x}_{\alpha} \overline{\bar{y}}_{1} x_{\alpha+1} x_{\alpha+2} \ldots x_{\alpha+\beta} \overline{\bar{y}}_{2} x_{\alpha+\beta+1} \ldots x_{\alpha+\beta+\gamma} \tilde{y}_{1} x_{\alpha+\beta+\gamma+1} x_{\alpha+\beta+\gamma+2} \ldots \\
\ldots x_{\alpha+\beta+\gamma+\delta} \tilde{y}_{2} x_{\alpha+\beta+\gamma+\delta+1} \ldots x_{\alpha+\beta+\gamma+\delta+\eta} \equiv 0 \quad(\bmod . \operatorname{Id}(\mathcal{V})) .
\end{gathered}
$$

Proof. Let $\lambda=(3,2) \vdash 5$ and $\mu=\left(1^{n-5}\right) \vdash n-5$ and let $M_{\lambda} \widehat{\otimes} M_{\mu}$ be the $S_{n}$ module outer tensor product of the $S_{5}$-module $M_{\lambda}$ and of the $S_{n-5}$-module $M_{\mu}$.

Consider the polynomials

$$
\begin{gathered}
y_{1} \bar{x}_{1} \bar{x}_{2} \ldots \bar{x}_{\alpha} \overline{\bar{y}}_{1} x_{\alpha+1} x_{\alpha+2} \ldots x_{\alpha+\beta} \overline{\bar{y}}_{2} x_{\alpha+\beta+1} \ldots x_{\alpha+\beta+\gamma} \tilde{y}_{1} x_{\alpha+\beta+\gamma+1} x_{\alpha+\beta+\gamma+2} \ldots \\
\ldots x_{\alpha+\beta+\gamma+\delta} \tilde{y}_{2} x_{\alpha+\beta+\gamma+\delta+1} \ldots x_{\alpha+\beta+\gamma+\delta+\eta}
\end{gathered}
$$

obtained from the two modules $M_{\lambda}$ and $M_{\mu}$. From remark after Lemma 2 these polynomials are identities of the variety $\mathcal{V}$. Let substitute $y_{1}$ with $y_{1}+z_{1} z_{2}$ and we are done.

Let's now examine some consequences of the previous Lemma.

In (11) let substitute $y_{1}$ with $y_{1}+z_{3}, y_{2}$ with $y_{2}+z_{4}$, and multilinearize. For simplicity we will not write the $x_{i}^{\prime} s$, then, modulo $\operatorname{Id}(\mathcal{V})$, we obtain

$$
\begin{gathered}
z_{1} z_{2} \ldots \overline{\bar{y}}_{1} \ldots \overline{\bar{y}}_{2} \ldots \tilde{z}_{3} \ldots \tilde{z}_{4} \ldots+z_{1} z_{2} \ldots \overline{\bar{z}}_{3} \ldots \overline{\bar{z}}_{4} \ldots \tilde{y}_{1} \ldots \tilde{y}_{2} \ldots+ \\
z_{1} z_{2} \ldots \overline{\bar{z}}_{3} \ldots \overline{\bar{y}}_{2} \ldots \tilde{y}_{1} \ldots \tilde{z}_{4} \ldots+z_{1} z_{2} \ldots \overline{\bar{y}}_{1} \ldots \overline{\bar{z}}_{4} \ldots \tilde{z}_{3} \ldots \tilde{y}_{2} \ldots \equiv 0 .
\end{gathered}
$$

In particular we have that

$$
\begin{gathered}
z_{1} z_{2} \ldots \overline{\bar{y}}_{1} \ldots \overline{\bar{y}}_{2} \ldots \tilde{z}_{3} \ldots \tilde{z}_{4} \ldots+z_{1} z_{2} \ldots \overline{\bar{z}}_{3} \ldots \overline{\bar{z}}_{4} \ldots \tilde{y}_{1} \ldots \tilde{y}_{2} \ldots+ \\
z_{1} z_{2} \ldots z_{3} \ldots y_{2} \ldots y_{1} \ldots z_{4} \ldots-z_{1} z_{2} \ldots y_{2} \ldots z_{3} \ldots y_{1} \ldots z_{4} \ldots- \\
z_{1} z_{2} \ldots z_{3} \ldots y_{2} \ldots z_{4} \ldots y_{1} \ldots+z_{1} z_{2} \ldots y_{2} \ldots z_{3} \ldots z_{4} \ldots y_{1} \ldots+ \\
z_{1} z_{2} \ldots y_{1} \ldots z_{4} \ldots z_{3} \ldots y_{2} \ldots-z_{1} z_{2} \ldots z_{4} \ldots y_{1} \ldots z_{3} \ldots y_{2} \ldots- \\
z_{1} z_{2} \ldots y_{1} \ldots z_{4} \ldots y_{2} \ldots z_{3} \ldots+z_{1} z_{2} \ldots z_{4} \ldots y_{1} \ldots y_{2} \ldots z_{3} \ldots \equiv 0 .
\end{gathered}
$$


If we alternate on $y_{1}, y_{2}$ and on $z_{3}, z_{4}$, we obtain that, modulo $\operatorname{Id}(\mathcal{V})$,

$$
\begin{gathered}
2 z_{1} z_{2} \ldots \overline{\bar{y}}_{1} \ldots \overline{\bar{y}}_{2} \ldots \tilde{z}_{3} \ldots \tilde{z}_{4} \ldots \equiv-2 z_{1} z_{2} \ldots \overline{\bar{z}}_{3} \ldots \overline{\bar{z}}_{4} \ldots \tilde{y}_{1} \ldots \tilde{y}_{2} \ldots+ \\
2 z_{1} z_{2} \ldots \tilde{z}_{3} \ldots \overline{\bar{y}}_{1} \ldots \overline{\bar{y}}_{2} \ldots \tilde{z}_{4} \ldots-2 z_{1} z_{2} \ldots \overline{\bar{y}}_{1} \ldots \tilde{z}_{3} \ldots \overline{\bar{y}}_{2} \ldots \tilde{z}_{4} \ldots- \\
2 z_{1} z_{2} \ldots \tilde{z}_{3} \ldots \overline{\bar{y}}_{1} \ldots \tilde{z}_{4} \ldots \overline{\bar{y}}_{2} \ldots+2 z_{1} z_{2} \ldots \overline{\bar{y}}_{1} \ldots \tilde{z}_{3} \ldots \tilde{z}_{4} \ldots \overline{\bar{y}}_{2} \ldots
\end{gathered}
$$

Let now consider the following substitutions $z_{i}=x_{\alpha+\beta+\gamma+\delta+\eta+i}$, for $i=1,2,3$, 4 , and let alternate on the variables $x_{1}, x_{2}, \ldots, x_{\alpha+\beta+\gamma+\delta+\eta+4}$, then it follows that, modulo $\operatorname{Id}(\mathcal{V})$,

$$
\begin{gathered}
\bar{x}_{1} \bar{x}_{2} \ldots \bar{x}_{\alpha+2} \tilde{y}_{1} \bar{x}_{\alpha+3} \ldots \bar{x}_{\alpha+\beta+2} \tilde{y}_{2} \bar{x}_{\alpha+\beta+3} \ldots \bar{x}_{\alpha+\beta+\gamma+\delta+\eta+4} \equiv \\
a \cdot \bar{x}_{1} \bar{x}_{2} \ldots \bar{x}_{\alpha+2} \tilde{y}_{1} \bar{x}_{\alpha+3} \ldots \bar{x}_{\alpha+\beta+\gamma+3} \tilde{y}_{2} \bar{x}_{\alpha+\beta+\gamma+4} \ldots \bar{x}_{\alpha+\beta+\gamma+\delta+\eta+4}+ \\
b \cdot \bar{x}_{1} \bar{x}_{2} \ldots \bar{x}_{\alpha+2} \tilde{y}_{1} \bar{x}_{\alpha+3} \ldots \bar{x}_{\alpha+\beta+\gamma+\delta+4} \tilde{y}_{2} \bar{x}_{\alpha+\beta+\gamma+\delta+5} \ldots \bar{x}_{\alpha+\beta+\gamma+\delta+\eta+4}+ \\
c \cdot \bar{x}_{1} \bar{x}_{2} \ldots \bar{x}_{\alpha+\beta+3} \tilde{y}_{1} \bar{x}_{\alpha+\beta+4} \ldots \bar{x}_{\alpha+\beta+\gamma+3} \tilde{y}_{2} \bar{x}_{\alpha+\beta+\gamma+4} \ldots \bar{x}_{\alpha+\beta+\gamma+\delta+\eta+4}+ \\
d \cdot \bar{x}_{1} \bar{x}_{2} \ldots \bar{x}_{\alpha+\beta+3} \tilde{y}_{1} \bar{x}_{\alpha+\beta+4} \ldots \bar{x}_{\alpha+\beta+\gamma+\delta+4} \tilde{y}_{2} \bar{x}_{\alpha+\beta+\gamma+\delta+5} \ldots \bar{x}_{\alpha+\beta+\gamma+\delta+\eta+4}+ \\
e \cdot \bar{x}_{1} \bar{x}_{2} \ldots \bar{x}_{\alpha+\beta+\gamma+4} \tilde{y}_{1} \bar{x}_{\alpha+\beta+\gamma+5} \ldots \bar{x}_{\alpha+\beta+\gamma+\delta+4} \tilde{y}_{2} \bar{x}_{\alpha+\beta+\gamma+\delta+5} \ldots \bar{x}_{\alpha+\beta+\gamma+\delta+\eta+4}
\end{gathered}
$$

where $a, b, c, d, e$ are equals to 1 or -1 according to the parity of the numbers $\alpha, \beta, \gamma, \delta, \eta$.

Let remark that from these identities it follows that it is possible to change the number of alternating variables between different alternating pair and we shall use this observation in the next proposition.

Proposition 6. If $\lambda=\left(2,2,1^{n-4}\right)$ then $m_{\lambda} \leq 3 m^{2}$.

Proof. Let $e_{T_{\lambda}} \in F S_{n}$ be the essential idempotent corresponding to the tableau $T_{\lambda}$, we shall identify $e_{T_{\lambda}}$ with the polynomial $e_{T_{\lambda}}\left(x_{1}, \ldots, x_{n}\right)=e_{T_{\lambda}} x_{1} \cdots x_{n}$ obtained by acting with $e_{T_{\lambda}}$ on the left normed monomial $x_{1} \cdots x_{n}$.

The polynomials corresponding to standard tableaux are of the following type

$$
\bar{x}_{1} \bar{x}_{2} \ldots \bar{x}_{\alpha} \tilde{x}_{1} x_{\alpha+1} x_{\alpha+2} \ldots x_{\alpha+\beta} \tilde{x}_{2} x_{\alpha+\beta+1} \ldots x_{\alpha+\beta+\gamma} .
$$

If $\alpha \geq m, \beta \geq m$ and $\gamma \geq m$ or $\alpha \geq m, \beta \geq m$ but $\gamma<m$ then, by Remark 4, we rewrite such polynomials as a linear combination of polynomials of the same type with $\beta<m$ and $\gamma<m$, then we have less than $m^{2}$ linearly independent polynomials.

If $\beta<m$ but $\gamma \geq m$ by the consequences of Lemma 7 , we rewrite such polynomials as a linear combinations of polynomials of the same type with $\beta \geq m$ or $\beta<m, \gamma<m$.

So, any polynomial is a linear combination of polynomials with $\alpha<m$ and $\beta<m$ or $\beta<m$ and $\gamma<m$ or $\alpha<m$ and $\gamma<m$.

It follows that, for $n \geq m, m_{\lambda} \leq 3 m^{2}$.

If $n<m$ then $m_{\lambda} \leq \bar{d}_{\lambda}<m^{2}$. Then, for any $n, m_{\lambda} \leq 3 m^{2}$ and we are done.

Now we are able to prove the following

Theorem 2. Let $\mathcal{V}$ be a variety of algebras satisfying the identity

$$
x(y z)=0 .
$$

If $c_{n}(\mathcal{V}) \leq C n^{\alpha}$ for some constant $C>0$ and $2<\alpha<3$, then $c_{n}(\mathcal{V}) \leq C_{1} n^{2}$ for some constant $C_{1}>0$. 
Proof. By Lemma 2, it follows that

$$
\begin{gathered}
\chi_{n}(\mathcal{V})=m_{(n)} \chi_{(n)}+m_{\left(1^{n}\right)} \chi_{\left(1^{n}\right)}+m_{(n-1,1)} \chi_{(n-1,1)}+m_{\left(2,1^{n-2}\right)} \chi_{\left(2,1^{n-2}\right)}+ \\
m_{(n-2,1,1)} \chi_{(n-2,1,1)}+m_{\left(3,1^{n-3}\right)} \chi_{\left(3,1^{n-3}\right)}+m_{(n-2,2)} \chi_{(n-2,2)}+m_{\left(2,2,1^{n-4}\right.} \chi_{\left(2,2,1^{n-4}\right)} .
\end{gathered}
$$

Since $\operatorname{deg} \chi_{(n)}=\operatorname{deg} \chi_{\left(1^{n}\right)}=1, \operatorname{deg} \chi_{(n-1,1)}=\operatorname{deg} \chi_{\left(2,1^{n-2}\right)}=n-1, \operatorname{deg} \chi_{\left(3,1^{n-3}\right)}=\frac{(n-1)(n-2)}{2}$ and $\operatorname{deg} \chi_{(n-2,2)}=\operatorname{deg} \chi_{\left(2,2,1^{n-4}\right)}=\frac{n(n-3)}{2}$, by recalling Proposition 3, Proposition 4, Proposition 5 and Proposition 6 we get

$$
\begin{gathered}
c_{n}(\mathcal{V}) \leq 2+2(n-1)^{2}+\frac{(n-1)(n-2)}{2}\left(5 m^{2}\right)+\frac{(n-1)(n-2)}{2}\left(5 m^{2}\right)+ \\
\frac{n(n-3)}{2} \bar{C}+\frac{n(n-3)}{2} 3 m^{2} \leq n^{2}\left(2+6.5 m^{2}+\bar{C}\right) .
\end{gathered}
$$

So there exists a costant $C_{1}$ such that $c_{n}(\mathcal{V}) \leq C_{1} n^{2}$.

[1] A. Berele, Homogeneous polynomial identities, Israel J. Math. 42 (1982), 258-272.

[2] V.Drensky, Representations of the symmetric group and varieties of linear algebras, Mat.Sb.(N.S.) 115 (1980), 98-115. Math.USSR-Sb.43 (1981), 85-100.

[3] V. Drensky, Relations for the cocharacter sequences of T-ideals, Proc. of the International Conference on Algebra Honoring A. Malcev, Contemp. Math. 131 (1992) (Part 2), 285-300.

[4] V. Drensky, "Free Algebras and PI-Algebras, Graduate course in algebra", Springer-Verlag Singapore, Singapore, 2000

[5] Giambruno, A.; Zaicev, M. On codimension growth of finitely generated associative algebras, Adv. Math. 140 (1998), 145-155. doi.org/10.1006/aima.1998.1766

[6] Giambruno, A.; Zaicev, M. Exponential codimension growth of P.I. algebras: an exact estimate, Adv. Math. 142 (1999), 221-243. doi.org/10.1006/aima.1998.1790

[7] A. Giambruno and M. Zaicev, Polynomial Identities and Asymptotic Methods, Mathematical Surveys and Monographs Vol. 122, American Mathematical Society, Providence, RI, 2005.

[8] G. James and A. Kerber, "The representation theory of the symmetric group", Encyclopedia of Mathematics and its Applications, Vol. 16, Addison-Wesley, London, 1981.

[9] A. Kemer, T-ideals with power growth of the codimensions are Specht, (Russian), Sibirskii Matematicheskii Zhurnal 19 (1978), 54-69; translation in Siberian Math. J. 19 (1978), 37-48.

[10] Mishchenko, S. P. On varieties of Lie algebras of intermediate growth, (Russian) VestsīAkad. Navuk BSSR Ser. Fìz.-Mat. Navuk 126 1987, no. 2, 42-45.

[11] Mishchenko, S. P. Lower bounds on the dimensions of irreducible representations of symmetric groups and of the exponents of the exponential of varieties of Lie algebras, (Russian) Mat. Sb. 187 (1996), 83-94; translation in Sb. Math. 187 (1996), 81-92.

[12] S. Mishchenko, A. Valenti, Varieties with at most quadratic growth, Israel J. Math., 178 (2010), 209228. doi.org/10.1007/s11856-010-0063-4

[13] S. Mishchenko, A. Valenti, On the growt of varieties of algebras, On the growth of varieties of algebras, "Groups, rings and group rings," Contemp. Math., Amer. Math Soc. ,Providence, RI, 499 (2009), 229243.

[14] S. P. Mishchenko, M. Zaicev, An example of a variety of linear algebras with the fractional polynomial growth, Vestnik of Moscow State Univ. Math. Mech. 2008 (1), 25-31.

[15] V. M. Petrogradsky, Growth of polynilpotent varieties of Lie algebras, and rapidly increasing entire functions, (Russian) Mat. Sb. 188 (1997), no. 6, 119-138; translation in Sb. Math. 188 (1997), no. 6, 913-931

[16] A. Regev, Existence of identities in $A \otimes B$, Israel J. Math. 11 (1972), 131-152.

[17] M. Zaicev, Integrality of exponents of growth of identities of finite-dimensional Lie algebras (Russian) Izv. Ross. Akad. Nauk Ser. Mat. 66 (2002), 23-48; translation in Izv. Math. 66 (2002), 463-487. No.2,2227.

[18] Zaicev, M. V.; Mishchenko, S. P. An example of a variety of Lie algebras with a fractional exponent Algebra, 11. J. Math. Sci. (New York) 93 (1999), no. 6, 977-982. 